\title{
ASF1B enhances migration and invasion of lung cancers cell via regulating the P53-mediated epithelial-mesenchymal transformation signaling pathway
}

\author{
Wei WANG*, Lina XIAO, Deng PAN, Lingzhi HU \\ Department of Respiratory and Critical Care Medicine, Tonglu First People's Hospital, Hangzhou, Zhejiang, China \\ ${ }^{*}$ Correspondence: wangweisun1981@163.com
}

Received August 18, 2021 / Accepted December 21, 2021

\begin{abstract}
Lung cancer is one of the most common malignant tumors in the world. Anti-silencing function 1B (ASF1B) has been demonstrated to play crucial roles in various tumors. However, the function of ASF1B in lung cancer remains to be addressed. Quantitative real-time polymerase chain reaction (qRT-PCR) and western blot assays demonstrated that ASF1B expression was upregulated in human lung cancer tissues and cells. High expression of ASF1B in lung cancer patients was associated with tumor stage and lymph node metastatic status and indicated a poor prognosis. The results of CCK- 8 and colony formation assays indicated that ASF1B promoted the proliferation of lung cancer cells. Moreover, ASF1B knockdown suppressed xenograft tumor growth and inhibited the levels of ASF1B and Ki-67. Transwell assay demonstrated that ASF1B promoted the migration and invasion of lung cancer cells. Importantly, mechanism analysis implied that upregulation of ASF1B decreased the expression of P53 and P21 while increasing the expression of Snail and Slug. Consistently, the knockdown of ASF1B led to the opposite results. Notably, P53 activation with Nutlin3 significantly weakened the epithelial-mesenchymal transformation (EMT) promotion effect of ASF1B, while P53 inhibition with pifithrin-a significantly enhanced the EMT promotion effect of sh-ASF1B. These data indicated that ASF1B exerts its oncogene function partially through the P53-mediated EMT signaling pathway. In conclusion, ASF1B promotes cell proliferation, migration, and invasion through modulating the P53-mediated EMT signaling pathway in lung cancer, suggesting that ASF1B may provide a promising target for the therapy of lung cancer.
\end{abstract}

Key words: ASF1B, lung cancer, migration, invasion, epithelial-mesenchymal transformation, P53

Lung cancer has become one of the most common and deadly cancers in the world $[1,2]$. Despite advances in early disease detection and treatment techniques in recent years, lung cancer patients are often diagnosed at the late stage, and their overall prognosis remains poor and is expected to continue to be the major disease burden in the first half of this century [3-5]. The 5-year survival rate for lung cancer patients is about $15 \%$ [6]. Metastases are a major cause of the high death rate from lung cancer, with advanced lung cancer often spreading to the brain, liver, or bones [7]. Epithelial-mesenchymal transformation (EMT) is an evolutionally conserved developmental process that is associated with carcinogenesis and imparts metastatic properties to cancer cells by enhancing cell migration, invasiveness, and resistance to apoptotic stimuli. Separation from the primary cells and invasion of the cancer cells into surrounding tissues may be due to a loss of intercellular adhesion and increased motility and aggressiveness [8]. Therefore, the identification of previously unidentified molecular targets leading to lung cancer development (especially metastasis) is not only biologically relevant but will also provide great clinical significance, possibly to promote the development of new drugs for lung cancer and suggest better treatment strategies.

Anti-silencing function 1 (ASF1) was originally identified in yeast and belongs to the chaperone protein of histone H3-H4 [9]. ASF1 plays a role in chromatin-based cellular DNA replication, DNA damage repair, and transcriptional regulation $[10,11]$. There are two main subtypes of ASF1, namely ASF1A and ASF1B. ASF1A is mainly involved in DNA repair and cell senescence, while ASF1B is preferentially involved in cell proliferation $[11,12]$. Overexpression of ASF1B resulted in different transcriptional characteristics consistent with increased cell proliferation and decreased cell apoptosis $[10,12]$. Armolle et al. found that high expression of ASF1B mRNA was associated with breast cancer clinical data and disease outcomes, and may be used as a new proliferation marker for breast cancer diagnosis and prognosis [13]. Recent studies have shown that ASF1B regulates the 
proliferation, migration, and apoptosis of cervical cancer cells by stabilizing CDK9 [14]. Studies have pointed out that ASF1B protein is involved in the occurrence and development of prostate cancer and clear cell renal cancer $[15,16]$. However, the biological function and mechanism of ASF1B in lung cancer are still unclear.

In the present study, we investigated the ASF1B expression levels in lung cancer tissues and cells. We explored the potential function of ASF1B in the proliferation, migration, and invasion of lung cancer cells, and the mechanism of ASF1B in the regulation of the P53-mediated EMT signaling pathway.

\section{Patients and methods}

Patients and tissues. This study was performed following the Declaration of Helsinki and approved by the Ethics Committees of Tonglu First People's Hospital with the written informed consent of all enrolled patients. 56 pairs of lung cancer tissues and matched adjacent normal tissues were collected from patients undergoing surgery in Tonglu First People's Hospital. None of them had received radiotherapy or chemotherapy before surgery.

Cell culture and transfection. Human lung cancer cell lines (A549, SPC-A1, H1975, H1229, and H460) and a normal human bronchial epithelial cell line (16HBE) were purchased from the American Type Culture Collection (ATCC, Manassas, VA, USA). All cell lines were maintained in Dulbecco's modified Eagle's medium (DMEM; Gibco; Thermo Fisher Scientific, Inc., Waltham, MA, USA). All medium supplemented with $10 \%$ fetal bovine serum (FBS, Gibco), $1 \%$ penicillin $(100 \mathrm{U} / \mathrm{ml}) /$ streptomycin $(100 \mu \mathrm{g} / \mathrm{ml})$ at $37^{\circ} \mathrm{C}$ in a humidified incubator with $5 \% \mathrm{CO}_{2}$. For overexpression and knockdown of ASF1B, the pcDNA3.1-ASF1B, pcDNA3.1-NC, shRNA targeting for ASF1B (sh-ASF1B\#1, sh-ASF1B\#2), and non-targeting shRNA (sh-NC) were obtained from GenePharma (Shanghai, China). Lipofectamine 3000 (Invitrogen, USA) was used for transfection according to the manufacturer's recommendations.

RNA extraction and qRT-PCR. Total RNA was extracted using TRIzol reagent (TaKaRa, Dalian, China) and reversely transcribed into complementary DNA (cDNA) using the PrimeScript ${ }^{\mathrm{TM}}$ RT Reagent Kit (TaKaRa) according to the manufacturer's instruction. qRT-PCR analysis was then performed using the SYBR Premix Ex Taq GC kit (Takara) in an ABI-7500 Real-Time PCR system (Applied Biosystems, USA) and the relative gene expression was analyzed using the $2^{-\triangle \Delta \mathrm{Ct}}$ method. The mRNA expression levels were normalized to GAPDH. The sequences of primers are summarized as follows: ASF1B, 5'-GATCAGCTTCGAGTGCAGTG-3' (forward), 5'-TGGTAGGTGCAGGTGATGAG-3' (reverse); GAPDH, 5'-CTGCACCACCAACTGCTTAG-3' (forward), 5'-AGGTCCACCACTGACACGTT-3' (reverse).

Western blot. Protein samples were extracted from tissues and cells using RIPA lysis buffer (Beyotime, Shanghai, China), resolved on $10 \%$ sodium dodecyl sulfate-polyacrylamide gel
(SDS-PAGE), and transferred to polyvinylidene difluoride (PVDF) membranes. The membranes were blocked with 5\% nonfat milk for $1 \mathrm{~h}$ and incubated with primary antibodies overnight at $4^{\circ} \mathrm{C}$. The primary antibodies are shown as follows: anti-ASF1B (1:500, \#ab276071, Abcam, Cambridge, UK), anti-E-cadherin (1:500, \#ab15148, Abcam), anti-Ncadherin (1:1000, \#ab76057, Abcam), anti-P53 (1:1000, \#ab154036, Abcam), anti-P21 (1:1000, \#ab109520, Abcam), anti-Snail (1:1000, \# ab216347, Abcam), anti-Slug (1:500, \#ab27568, Abcam), anti-GAPDH (1:500, \#ab8245, Abcam). Subsequently, membranes were incubated with HRP-conjugated goat anti-rabbit or anti-mouse IgG (1:2000, \#ab6721, \#ab97023, Abcam) for $1 \mathrm{~h}$ at room temperature. The band was visualized by the enhanced chemiluminescence (ECL) reagents (Pierce, USA) and analyzed by an imaging system (Bio-Rad, Hercules, USA).

Cell proliferation assay. For Cell Counting Kit- 8 (CCK-8) assay, $1 \times 10^{4}$ cells were plated into 96 -well plates and transfected as required. Cells were treated with $10 \mu \mathrm{l}$ of CCK-8 solution (Dojindo, Japan) for $2 \mathrm{~h}$ at $37^{\circ} \mathrm{C}$ according to the manufacturer's protocols. The cell viability was measured by the optical density value at $450 \mathrm{~nm}$ using a microplate reader (Bio-Rad, Hercules, CA, USA) at the specified time point ( $24 \mathrm{~h}, 48 \mathrm{~h}, 72 \mathrm{~h}$, and $96 \mathrm{~h}$ ) after transfection. Moreover, for colony formation assay, $1 \times 10^{3}$ cells were seeded into 6 -well plates and DMEM medium was changed every 3 days for 2 weeks until clones were visible. The colonies were washed by phosphate-buffered saline (PBS, Gibco) twice and fixed in paraformaldehyde (PFA, Sigma-Aldrich, St. Louis, USA) for $15 \mathrm{~min}$. Then the colonies were stained with $0.5 \%$ crystal violet (Beyotime) for $20 \mathrm{~min}$. Staining cells were washed in PBS, counted, and photographed using a light microscope (Olympus, Tokyo, Japan).

Tumor xenograft. The animal experiments were performed following the guideline for the care and use of laboratory animals with the approval of Tonglu First People's Hospital. The male BALB/c nude mice aged 4-6-weeks old were purchased from the Shanghai Experimental Animals Centre (Shanghai, China). The nude mice were randomly divided into two groups for the tumor formation assay. sh-NC or sh-ASF1B transfected A549 cells $\left(1 \times 10^{7}\right)$ were injected into the right flanks of nude mice ( $n=6 /$ group). The tumor size was detected using vernier calipers every 3 days and calculated by the formula: tumor volume $\left(\mathrm{mm}^{3}\right)=$ length $(\mathrm{mm}) \times$ width $(\mathrm{mm})^{2} \times 0.5$. Mice were euthanized 28 days after inoculation, and tumors were excised and weighed. The tumor tissues were fixed for subsequent immunohistochemistry (IHC) assay.

IHC assay. The tumor tissues were fixed with 4\% PFA (Sigma-Aldrich), embedded in paraffin, and sliced into 4 $\mu \mathrm{m}$ sections. After that, the sections were incubated with primary antibody ASF1B and Ki-67 (Abcam) overnight at $4{ }^{\circ} \mathrm{C}$. Next, the sections were incubated with the corresponding secondary antibody for $1 \mathrm{~h}$ at room temperature. The immunocomplexes were visualized using 3,3'-diamino- 
benzidine tetrahydrochloride (DAB, Solarbio), and counterstained by hematoxylin. The samples were observed under a light microscope (Olympus, Japan).

Transwell assay. $4 \times 10^{4}$ cells were suspended in the $500 \mu \mathrm{l}$ of serum-free medium and placed in the upper chamber $(8$ $\mu \mathrm{m}$ pore size; Corning, Tewksbury, MA, USA) with uncoated (for cell migration assay) or pre-coated with Matrigel (for cell invasion assay; BD Biosciences, MA), while $400 \mu \mathrm{l}$ of DMEM medium containing 10\% FBS were added in the lower chambers. Afterward, cells in the lower chamber were fixed by $4 \%$ paraformaldehyde at $24 \mathrm{~h}$ post-incubation. Then, cells were darkly stained $0.5 \%$ crystal violet for $30 \mathrm{~min}$. In the endpoint, cells were counted and photographed under a microscope (Olympus).

Statistical analysis. The data were processed utilizing Statistical product (SPSS, version 20.0) and displayed as mean \pm standard deviation (SD). Student's t-test was used for two-group comparisons, and one-way analysis of variance was used for that of more than two group comparisons. All experiments were performed at least three times. A p-value $<0.05$ was defined as statistically significant.

\section{Results}

ASF1B is highly expressed in lung cancer and has a poor prognosis. According to UALCAN (http://UALCAN. path.uab.edu/analysis.html) data [17], ASF1B expression is significantly increased in lung cancer compared to normal lung tissue (Figure 1A). Additionally, ASF1B expression was associated with tumor stage and lymph node metastatic status (Figures 1B, 1C). Survival curves from Kaplan-Meier Plotter (http://kmplot.com/analysis/) [18] suggest that high expression of ASF1B in lung cancer patients indicated a poor prognosis $(\mathrm{HR}=1.26 ; 95 \% \mathrm{CI}, 1.05-1.52$; log-rank $\mathrm{p}=0.015$; Figure 1D). Similarly, the further qRT-PCR analysis indicated that ASF1B mRNA expression was obviously upregulated in lung cancer tissues compared to the corresponding adjacent normal tissues (Figure 1E). Consistently, western blot analysis demonstrated that ASF1B protein level was significantly increased lung cancer tissues (Figure 1F). Then, we used 16HBE, A549, SPC-A1, H1975, H1229, and H460 cell lines to evaluate the role of ASF1B in lung cancer cell lines. ASF1B expression at the mRNA and protein levels was examined via qRT-PCR and western blot assays. We observed that ASF1B was overexpressed in the lung cancer cell lines compared to the 16HBE cell line (Figures 1G, 1H). In addition, ASF1B was the least expressed in SPC-A1 cells and relatively higher in A549 cells (Figures 1G, 1H). Therefore, the SPC-A1 cell line was selected as a candidate cell line for ASF1B overexpression, while the A549 cell line was selected as a candidate cell line for ASF1B knockdown in subsequent experiments. Taken together, these data suggest that ASF1B is upregulated in lung cancer and associated with the prognosis of patients.

ASF1B enhances the proliferation of lung cancer cells in vitro. To characterize the function of ASF1B in lung cancer cells, we overexpressed the ASF1B in SPC-A1 cells and knockdown ASF1B using two shRNAs in A549 cells. The transfected efficiencies were confirmed by qRT-PCR and western blot assays. As illustrated in Figures 2A and 2B, ASF1B expression was markedly increased in the ASF1B group compared to the Vector group, which indicated that overexpression of ASF1B is successful in SPC-A1 cells. Moreover, each shRNA could effectively decrease ASF1B expression, sh-ASF1B\#1 displayed the better knockdown efficiency in A549 cells therefore, it was selected for further investigations (Figures 2A, 2B). After that, the CCK-8 assay showed that the overexpression of ASF1B promoted the cell viability of SPC-A1 cells while the downregulation of ASF1B prominently suppressed the cell viability of A549 cells (Figure 2C). Moreover, colony formation analysis revealed that elevated ASF1B expression resulted in an increase in colony number in SPC-A1 cells, whereas ASF1B knockdown lead to fewer colonies in the A549 cells (Figure 2D). These results suggested that ASF1B can promote the proliferation of lung cancer cells in vitro.

Knockdown of ASF1B hinders the growth of lung cancer xenograft tumors in vivo. To further validate the significance of ASF1B on tumor growth in vivo, a xenograft tumor model was constructed. As revealed in Figures 3A-3C, ASF1B knockdown markedly decreased the tumor size, tumor volume, and tumor weight of mice compared with the sh-NC group. Moreover, the results of the IHC assay indicated that ASF1B and Ki-67 levels were obviously decreased in tumor tissues from the sh-ASF1B group compared to the sh-NC group (Figure 3D). All observations in vivo demonstrated that the knockdown of ASF1B inhibited tumorigenesis of lung cancer.

ASF1B enhances the migration and invasion of lung cancer cells. Transwell assay was performed to analyze the migration and invasion of lung cancer cells. The data indicated that upregulation of ASF1B significantly increased the migration and invasion abilities of SPC-A1 cells while downregulation of ASF1B observed the opposite results in A549 cells (Figures 4A, 4B). Subsequent western blot analysis implied that overexpression of ASF1B significantly reduced the expression of epithelial marker E-cadherin, whereas increased the expression level of mesenchymal marker $\mathrm{N}$-cadherin in SPC-A1 cells (Figures 4C, 4D). However, the knockdown of ASF1B exerted an opposite effect in A549 cells (Figures 4C, 4D). These data concluded that ASF1B enhances the migratory and invasive capabilities of lung cancer cells.

ASF1B may promote migration and invasion of lung cancer cells by regulating the P53-mediated EMT signaling pathway. To explore the potential mechanism by which ASF1B regulates the progression of lung cancer, we detected the expression changes of relevant proteins by western blot. As shown in Figure 5A, the expression levels of P53 and P21 were obviously reduced, while the expression levels of Snail and Slug were significantly increased in the ASF1B overexpression group of SPC-A1 cells. However, the opposite effects 
A

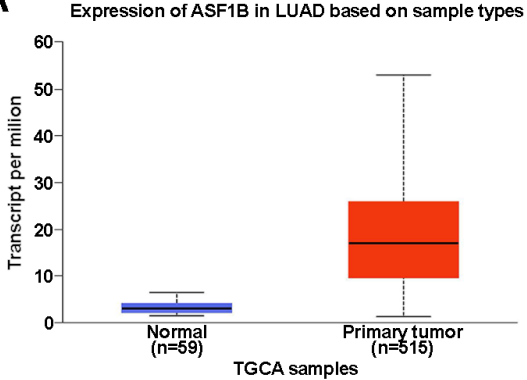

C

Expression of ASF1B in LUAD based on nodal metastasis
StatuS

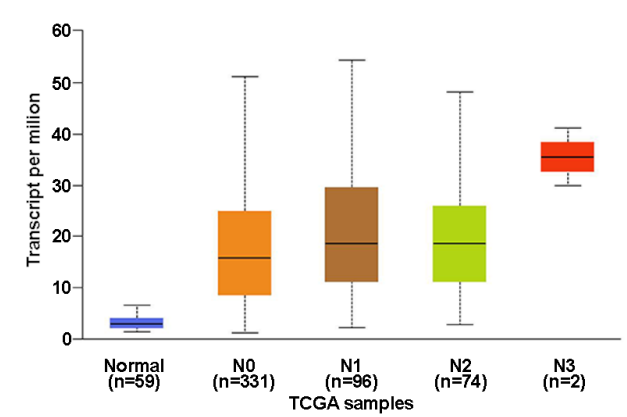

E

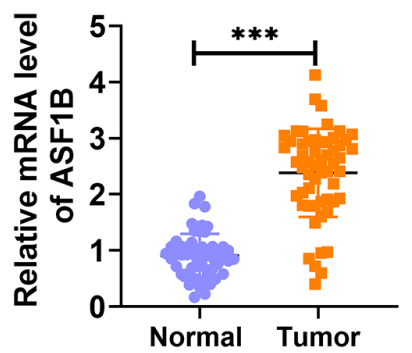

G

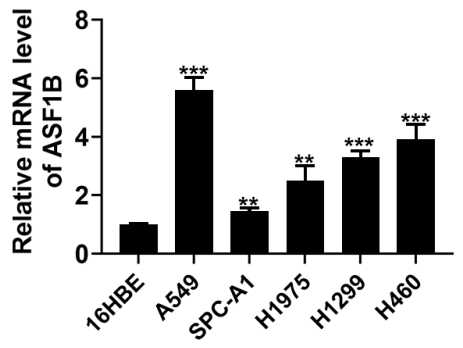

$\mathrm{H}$
B Expression of ASF1B in LUAD based on individual cancer

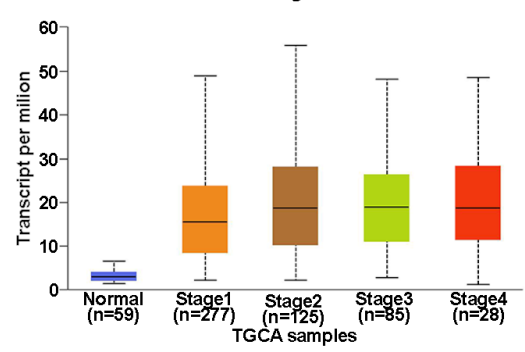

D

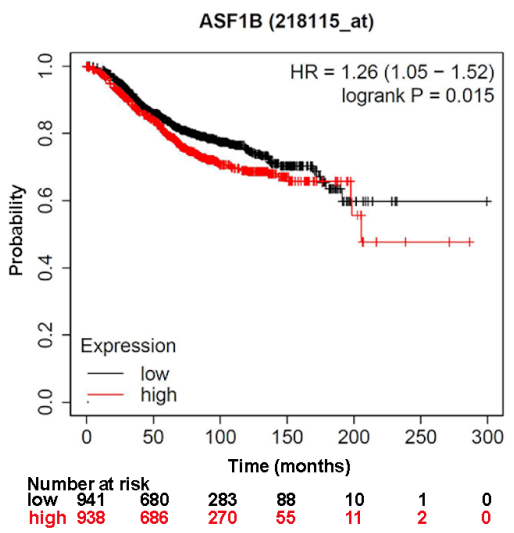

F

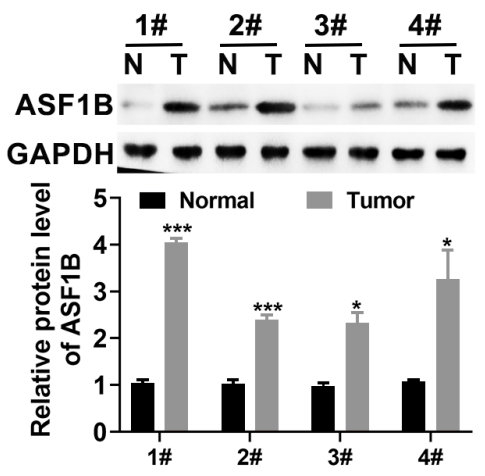

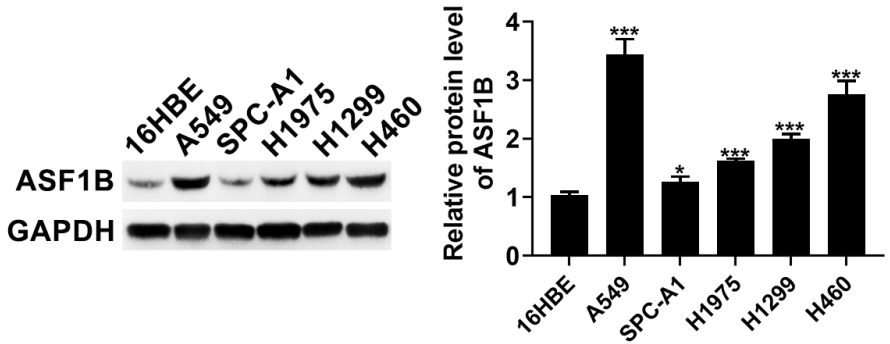

Figure 1. ASF1B is highly expressed in lung cancer and has a poor prognosis. A) UALCAN database displays the expression of ASF1B in lung adenocarcinoma (LUAD) based on sample type. B) UALCAN database displays the expression of ASF1B in LUAD based on individual cancer stages. C) UALCAN database displays the expression of ASF1B in LUAD based on nodal metastasis status (N0, no regional lymph node metastasis; N1, metastases in 1-3 axillary lymph nodes; N2, metastases in 4-9 axillary lymph nodes; N3, metastases in 10 or more axillary lymph nodes). D) Effect of ASF1B mRNA expression in lung cancer tissues on patient overall survival was assessed based on the database of the Kaplan-Meier Plotter platform. E) The mRNA expression of ASF1B in 56 paired lung cancer tissues and matched adjacent normal tissues were determined by qRT-PCR assay. F) The protein level of ASF1B in lung cancer tissues $(n=4)$ and adjacent normal tissues $(n=4)$ was detected by western blot assay. $G)$ The mRNA expression of ASF1B in the normal human bronchial epithelioid cells (16HBE) and in lung cancer cell lines (A549, SPC-A1, H1975, H1229, and H460) was determined by qRTPCR assay. H) The protein level of ASF1B in the normal human bronchial epithelioid cells (16HBE) and in lung cancer cell lines (A549, SPC-A1, H1975, H1229, and H460) was determined by western blot assay. Data are shown as mean $\pm \mathrm{SD} ;{ }^{\star} \mathrm{p}<0.05$, ${ }^{\star *} \mathrm{p}<0.01$, and ${ }^{\star * *} \mathrm{p}<0.001$. 
A

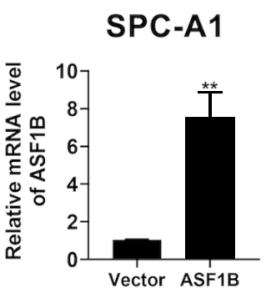

B

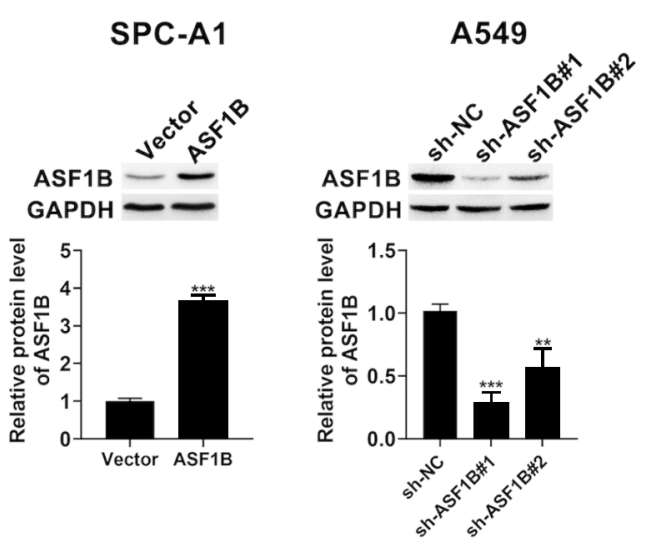

C

SPC-A1

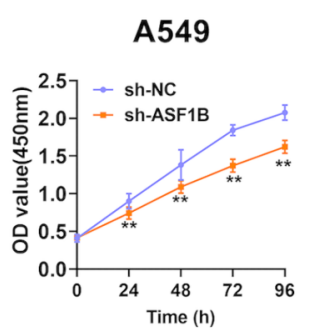

D

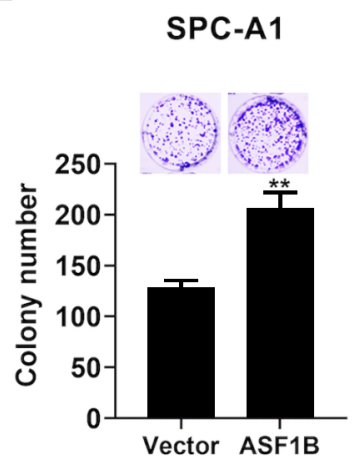

A549

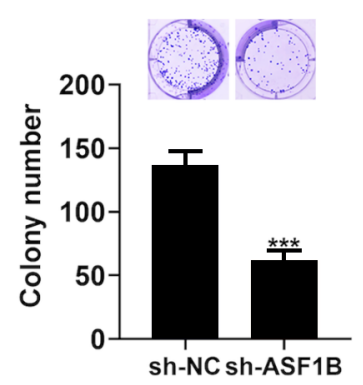

Figure 2. ASF1B enhances the proliferation of lung cancer cells in vitro. A) The mRNA expression of ASF1B in SPC-A1 and A549 cells was detected by the qRT-PCR assay. B) The protein level of ASF1B in SPC-A1 and A549 cells was detected by western blot assay. C) CCK-8 assay was used to determine the cell viabilities of SPC-A1 and A549 cells. D) Colony formation assay was used to detect the number of cell clones in SPC-A1 and A549 cells. Data are shown as mean $\pm S D ;{ }^{*} p<0.05,{ }^{* *} \mathrm{p}<0.01$, and ${ }^{* * *} \mathrm{p}<0.001$.

\section{A}

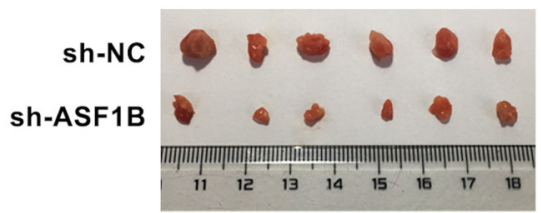

C

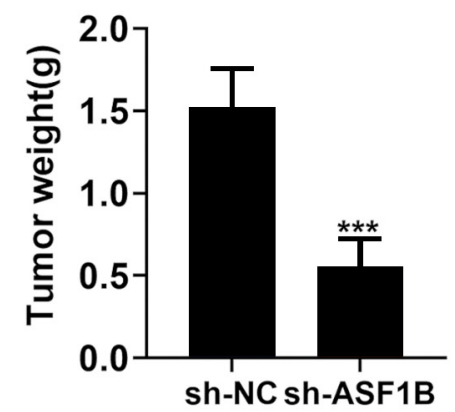

B
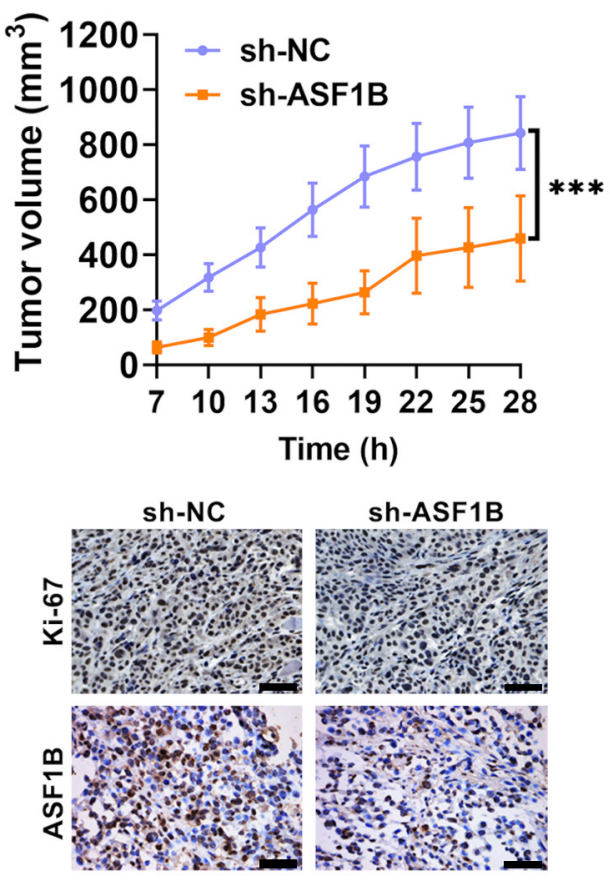

Figure 3. Knockdown of ASF1B hinders the growth of lung cancer xenograft tumors in vivo. A) Photographs of tumors excised from the mice injected with A549 cells transfected with sh-NC or sh-ASF1B. B) Tumor growth curves of mice injected with A549 cells. C) Tumor weight of mice injected with A549 cells. D) The levels of ASF1B and Ki-67 in tumors from mice injected with A549 cells were evaluated by IHC staining (Scale bar $200 \mu \mathrm{m}$, magnification 100 $)$. Data are shown as mean $\pm \mathrm{SD} ;{ }^{*} \mathrm{p}<0.05,{ }^{* *} \mathrm{p}<0.01$, and ${ }^{* * *} \mathrm{p}<0.001$. 
A
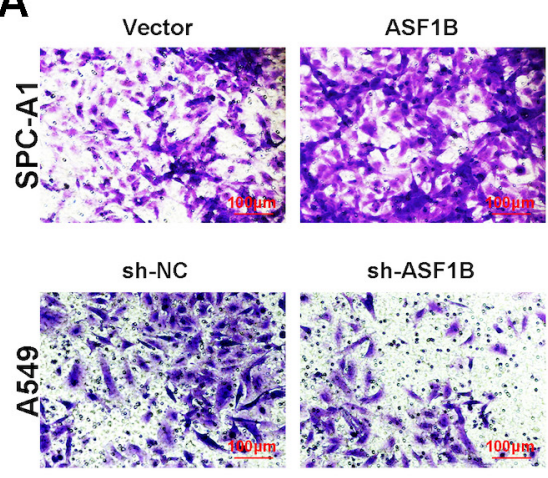

C
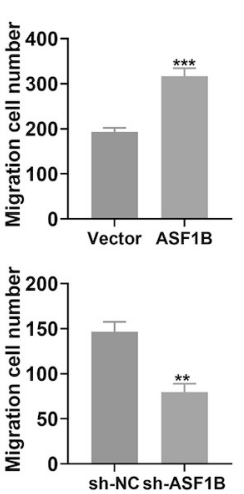

SPC-A1
B
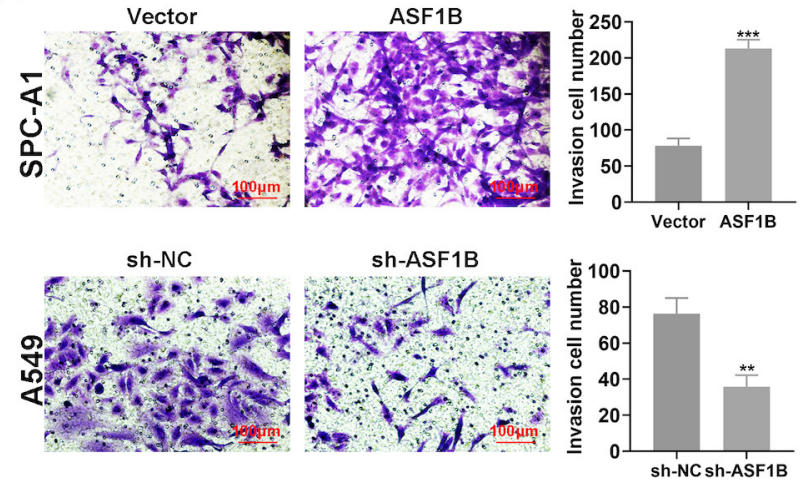

D
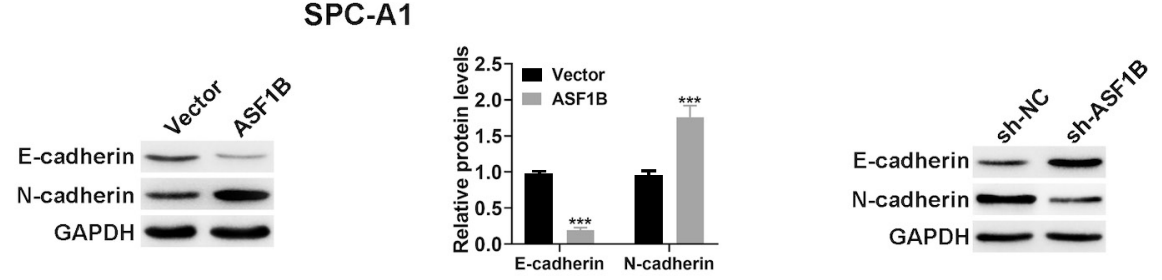

A549

Figure 4. ASF1B enhances the migration and invasion of lung cancer cells. A, B) Transwell assay was used to detect the migration and invasion capabilities of SPC-A1 and A549 cells (Scale bar $100 \mu \mathrm{m}$, magnification 200×). C, D) The levels of E-cadherin and N-cadherin in SPC-A1 and A549 cells were measured by western blot analysis. Data are shown as mean $\pm \mathrm{SD} ;{ }^{\star} \mathrm{p}<0.05,{ }^{* *} \mathrm{p}<0.01$, and ${ }^{\star * *} \mathrm{p}<0.001$.

were observed when ASF1B was knocked down. These data indicated that ASF1B might regulate lung cancer cell migration and invasion via the $\mathrm{P} 53$ signaling pathway. Furthermore, we assessed the effect of the presence or absence of Nutlin3 (a P53 activator) on the ASF1B-overexpressing cells. Unsurprisingly, Nutlin3 treatment reversed the ASF1B overexpression-induced increases of Snail and Slug and induced reduction of P53 and P21 (Figure 5A). Moreover, the effect of the presence or absence of pifithrin- $\alpha$ (a P53 inhibitor) on the ASF1B-knockdown cells. The data indicated that pifithrin- $\alpha$ treatment reversed the ASF1B-knockdown-induced reduction of Snail and Slug and -induced increases of P53 and P21 (Figure 5B). Together, we deduced that ASF1B induces lung cancer cell migration and invasion via regulating the P53-mediated EMT signaling pathway.

\section{Discussion}

In this study, we demonstrated that ASF1B expression was statistically upregulated in lung cancer tissues, and the patients with high ASF1B expression predicted poorer survival. Functional studies indicated that ASF1B dramatically promoted lung cancer cell proliferation, migration, and invasion in vitro, as well as tumor growth in vivo. These data revealed that $\mathrm{ASF} 1 \mathrm{~B}$ is a novel tumor oncogene and may be a new target for the treatment and prognosis of lung cancer.

At present, the role of ASF1B in cancer has been gradually explored. A recent study has shown that the increased expres- sion of ASF1B and the poor prognosis of patients with malignant mesothelioma, ASF1B may regulate the tumor microenvironment and EMT to induce differentiation of the sarcomalike phenotype (https://doi.org/10.21203/rs.3.rs-146040/v1; published preliminary reports). A newly published study has shown that the upregulation of ASF1B in lung cancer tumor tissues is associated with poorer overall survival and progression-free survival, as well as with advanced stage and tumor progression [19]. Consistently, an analysis of TCGA data in this study showed that ASF1B expression was significantly elevated in lung cancer tissues, correlated with tumor stage and lymph node metastatic status, and patients with high ASF1B expression had a poor prognosis. Further experimental results also confirmed the abnormal increase of ASF1B in lung cancer tissues and cells. These data suggest that ASF1B may be involved in cancer progression. Previous studies have confirmed that ASF1B can regulate tumor cell proliferation, cycle progression, apoptosis, migration, invasion, EMT, tumor microenvironment, and growth in various types of cancer [14-16, 19]. Here, we investigated the function of ASF1B in lung cancer for the first time. As in other cancers, our findings confirmed the protumor effects of ASF1B in lung cancer, in which ASF1B promoted the proliferation, migration and invasion of lung cancer cells in vitro, and the depletion of ASF1B decreased tumor growth in vivo.

Accumulating studies have shown that EMT is an important biological process for the migration and invasion of malignant cells of epithelial origin [20]. When the cell under- 
A SPC-A1
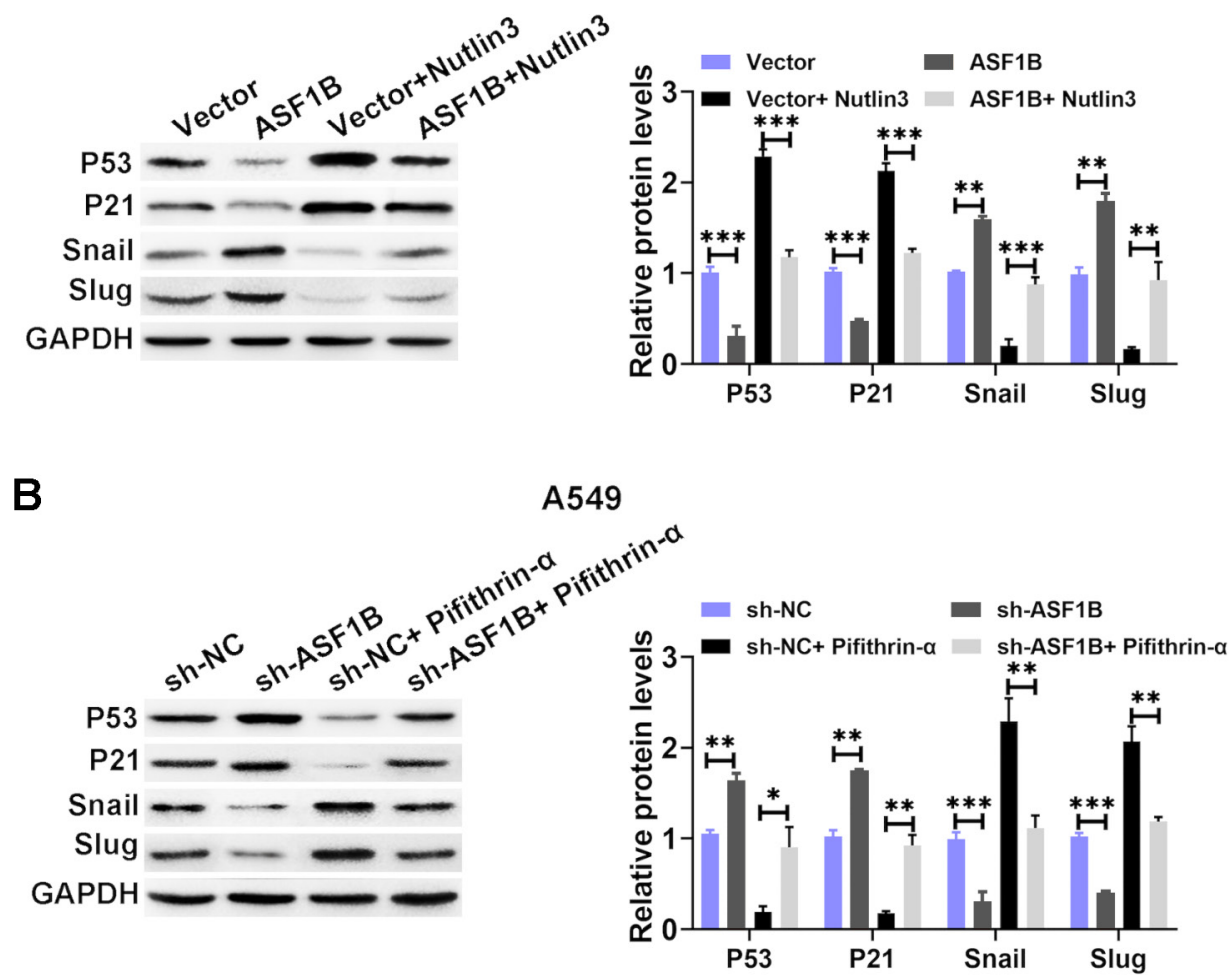

Figure 5. ASF1B may promote migration and invasion of lung cancer cells by regulating the P53-mediated EMT signaling pathway. A) The levels of P53, P21, Snail, and Slug in SPC-A1 cells were determined by western blot assay. Western blot analysis of the indicated proteins after treatment with Nutlin3 $(10 \mu \mathrm{M})$ for $72 \mathrm{~h} . \mathrm{B})$ The levels of P53, P21, Snail, and Slug in A549 cells were determined by western blot assay. Western blot analysis of the indicated proteins after treatment with pifithrin- $\alpha(15 \mu \mathrm{M})$ for $72 \mathrm{~h}$. Data are shown as mean $\pm \mathrm{SD} ;{ }^{*} \mathrm{p}<0.05,{ }^{* *} \mathrm{p}<0.01$, and ${ }^{* * *} \mathrm{p}<0.001$.

goes EMT, the epithelial-like characteristics of the cell gradually weaken, while the interstitial characteristics gradually appear, the polarity disappears, and the adhesion is weakened, thus gradually showing an infiltrating phenotype $[8,21]$. It is worth noting that the metastasis of tumors with EMT characteristics is upregulated by transcription factors such as Snail and Slug, which leads to the downregulation of the epithelial marker E-cadherin protein. At the same time, interstitial tumor cell markers, such as $\mathrm{N}$-cadherin, are upregulated, endowing tumor cells with invasion and spreading capabilities $[8,20,22]$. At present, ASF1B promotes the EMT process in lung cancer cells, which is characterized by increased expression of N-cadherin, Snail, and Slug, while decreased expression of E-cadherin. Taken together, these data suggest that ASF1B is involved in the EMT process and affects the migration and invasion of lung cancer cells.

ASF1B has been reported to exert its carcinogenic effects through diverse mechanisms. ASF1B has been reported to promote tumor progression in prostate cancer and clear cell renal cell carcinoma through activation of the PI3K/ Akt pathway $[15,16]$. Moreover, it has been reported that ASF1B promoted cervical cancer tumor growth by forming a stable complex with cyclin-dependent kinase 9 (CDK9) and promoted the proteasome stability of CDK9 [14]. Recently, a study showed that most of the genes inhibited by ASF1B were enriched in apoptosis and cell death-related pathways (such as the P53 signaling pathway) [12]. P53 is thought to be an important tumor suppressor that plays vital roles in multiple crucial biological processes, including cell growth, cell cycle, apoptosis, DNA repair, and EMT [22-26]. In addition, P53 is usually inactivated during lung cancer progression, and promoting the P53 pathway can reduce the growth and metastasis of lung cancer cells $[27,28]$. However, it is not clear whether ASF1B can regulate the P53 signaling pathway. Notably, our data herein a new regulatory mechanism to depict the interaction of ASF1B in the carcinogenesis and metastasis of lung cancer. In the present study, for the first time, we discovered that ASF1B inhibited the levels of P53 and the downstream target protein P21, and promoted the levels of Snail and Slug in lung cancer. In terms of the mechanisms underlying ASF1B-facilitated the EMT of lung cancer cells, P53 activation with Nutlin3 significantly weakened the EMT promotion effect of ASF1B, while P53 inhibition with pifithrin- $\alpha$ significantly enhanced the EMT promotion effect of sh-ASF1B. According to previous studies, P53 directly affects the transcription of transfer-related genes 
that regulate cell movement, adhesion, and EMT $[25,26,30]$. In addition, the P53 signaling pathway affects EMT-related transcription factors such as Snail and Slug, which regulate E-cadherin and $\mathrm{N}$-cadherin, etc. [27, 29-31]. Thus, we propose that the P53-mediated EMT signaling pathway is partially required for the role of ASF1B in cell migration and invasion of lung cancer. However, it cannot be ruled out that other downstream molecules of ASF1B are involved in this complex biological process. Our study revealed a new regulatory relationship between $\mathrm{ASF} 1 \mathrm{~B}$ and $\mathrm{P} 53$, and the exact mechanism of whether ASF1B is involved in P53 degradation requires further exploration.

In conclusion, the results of the present study demonstrated that ASF1B expression was upregulated in lung cancer tissues and cell lines. ASF1B enhanced proliferation, migration, invasion of lung cancer cells in vitro, the knockdown of ASF1B inhibited the in vivo tumor growth. Mechanistic experiments indicate that the P53-mediated EMT signaling pathway may be a possible contributor to the carcinogenic effect of ASF1B. Thus, ASF1B may serve as a potential therapeutic target for patients with lung cancer.

\section{References}

[1] FERLAY J, COLOMBET M, SOERJOMATARAM I, MATHERS C, PARKIN DM et al Estimating the global cancer incidence and mortality in 2018: GLOBOCAN sources and methods. International Journal of Cancer 2019; 144: 1941-1953. https://doi.org/10.1002/ijc.31937

[2] BRAY F, FERLAY J, SOERJOMATARAM I, SIEGEL RL, TORRE LA et al Global cancer statistics 2018: GLOBOCAN estimates of incidence and mortality worldwide for 36 cancers in 185 countries. CA: A Cancer Journal for Clinicians 2018; 68: 394-424. https://doi.org/10.3322/caac.21492

[3] CHENG TY, CRAMB SM, BAADE PD, YOULDEN DR, NWOGU $C$ et al The International Epidemiology of Lung Cancer: Latest Trends, Disparities, and Tumor Characteristics. J Thorac Oncol 2016; 11: 1653-1671. https://doi. org/10.1016/j.jtho.2016.05.021

[4] AGUSTONI F, SUDA K, YU H, REN S, RIVARD CJ et al EGFR-directed monoclonal antibodies in combination with chemotherapy for treatment of non-small-cell lung cancer: an updated review of clinical trials and new perspectives in biomarkers analysis. Cancer Treat Rev 2019; 72: 15-27. https://doi.org/10.1016/j.ctrv.2018.08.002

[5] WU SG, SHIH JY. Management of acquired resistance to EGFR TKI-targeted therapy in advanced non-small cell lung cancer. Mol Cancer 2018; 17: 38. https://doi.org/10.1186/ s12943-018-0777-1

[6] HEIST RS, ENGELMAN JA. SnapShot: non-small cell lung cancer. Cancer Cell 2012; 21: 448.e2. https://doi. org/10.1016/j.ccr.2012.03.007

[7] MILOVANOVIC IS, STJEPANOVIC M, MITROVIC D. Distribution patterns of the metastases of the lung carcinoma in relation to histological type of the primary tumor: An autopsy study. Annals of thoracic medicine 2017; 12: 191-198. https://doi.org/10.4103/atm.ATM_276_16
[8] MITTAL V. Epithelial Mesenchymal Transition in Tumor Metastasis. Annu Rev Pathol 2018; 13: 395-412. https://doi. org/10.1146/annurev-pathol-020117-043854

[9] MIKNIS GF, STEVENS SJ, SMITH LE, OSTROV DA, CHURCHILL ME. Development of novel Asf1-H3/H4 inhibitors. Bioorg Med Chem Lett 2015; 25: 963-968. https:// doi.org/10.1016/j.bmcl.2014.11.067

[10] PENG H, NOGUEIRA ML, VOGEL JL, KRISTIE TM. Transcriptional coactivator HCF-1 couples the histone chaperone Asf1b to HSV-1 DNA replication components. Proceedings of the National Academy of Sciences 2010; 107: 2461. https:// doi.org/10.1073/pnas.0911128107

[11] MESSIAEN S, GUIARD J, AIGUEPERSE C, FLINIAUX I, TOURPIN $S$ et al Loss of the histone chaperone ASF1B reduces female reproductive capacity in mice. Reproduction 2016; 151: 477-489. https://doi.org/10.1530/REP-15-0327

[12] PAUL PK, RABAGLIA ME, WANG CY, STAPLETON DS, LENG $\mathrm{N}$ et al Histone chaperone ASF1B promotes human $\beta$-cell proliferation via recruitment of histone H3.3. Cell Cycle 2016; 15: 3191-3202. https://doi.org/10.1080/15384101.2 016.1241914

[13] CORPET A, DE KONING L, TOEDLING J, SAVIGNONI A, BERGER F et al Asflb, the necessary Asf1 isoform for proliferation, is predictive of outcome in breast cancer. EMBO J 2011; 30: 480-493. https://doi.org/10.1038/emboj.2010.335

[14] LIU X, SONG J, ZHANG Y, WANG H, SUN H et al ASF1B promotes cervical cancer progression through stabilization of CDK9. Cell Death Dis 2020; 11: 705. https://doi. org/10.1038/s41419-020-02872-5

[15] HAN G, ZHANG X, LIU P, YU Q, LI Z et al Knockdown of anti-silencing function $1 \mathrm{~B}$ histone chaperone induces cell apoptosis via repressing PI3K/Akt pathway in prostate cancer. Int J Oncol 2018; 53: 2056-2066. https://doi.org/10.3892/ ijo. 2018.4526

[16] JIANGQIAO Z, TAO Q, ZHONGBAO C, XIAOXIONG M, LONG $\mathrm{Z}$ et al Anti-silencing function $1 \mathrm{~B}$ histone chaperone promotes cell proliferation and migration via activation of the AKT pathway in clear cell renal cell carcinoma. Biochem Biophys Res Commun 2019; 511: 165-172. https://doi. org/10.1016/j.bbrc.2019.02.060

[17] CHANDRASHEKAR DS, BASHEL B, BALASUBRAMANYA SAH, CREIGHTON CJ, PONCE-RODRIGUEZ I et al. UALCAN: A Portal for Facilitating Tumor Subgroup Gene Expression and Survival Analyses. Neoplasia 2017; 19: 649658. https://doi.org/10.1016/j.neo.2017.05.002

[18] NAGY Á, LÁNCZKY A, MENYHÁRT O, GYÖRFFY B. Validation of miRNA prognostic power in hepatocellular carcinoma using expression data of independent datasets. Scientific Reports 2018; 8: 9227. https://doi.org/10.1038/ s41598-018-27521-y

[19] FENG Z, ZHANG J, ZHENG Y, WANG Q, MIN X et al Elevated expression of ASF1B correlates with poor prognosis in human lung adenocarcinoma. Per Med 2021; 18: 115-127. https://doi.org/10.2217/pme-2020-0112

[20] PASTUSHENKO I, BLANPAIN C. EMT Transition States during Tumor Progression and Metastasis. Trends Cell Biol 2019; 29: 212-226. https://doi.org/10.1016/j.tcb.2018.12.001 
[21] BRABLETZ T, KALLURI R, NIETO MA, WEINBERG RA. EMT in cancer. Nat Rev Cancer 2018; 18: 128-134. https:// doi.org/10.1038/nrc.2017.118

[22] WU Y, ZHOU BPJCA, MIGRATION. Snail: more than EMT. Cell Adh Migr 2010; 4: 199-203. https://doi.org/10.4161/ cam.4.2.10943

[23] WEE KB, AGUDA BD. Akt versus p53 in a Network of Oncogenes and Tumor Suppressor Genes Regulating Cell Survival and Death. Biophys J 2006; 91: 857-865. https://doi. org/10.1529/biophysj.105.077693

[24] LOZANO G. Restoring p53 in cancer: the promises and the challenges. Journal of Molecular Cell Biol 2019; 11: 615-619. https://doi.org/10.1093/jmcb/mjz063

[25] ROSZKOWSKA KA, GIZINSKI S, SADY M, GAJEWSKI Z, OLSZEWSKI MB. Gain-of-Function Mutations in p53 in Cancer Invasiveness and Metastasis. Int J Mol Sci 2020; 21 : 1334. https://doi.org/10.3390/ijms21041334

[26] JANIC A, VALENTE LJ, WAKEFIELD MJ, DI STEFANO L, MILLA L et al. DNA repair processes are critical mediators of p53-dependent tumor suppression. Nature Medicine 2018; 24: 947-953. https://doi.org/10.1038/s41591-018-0043-5
[27] TAKAHASHI T, NAU MM, CHIBA I, BIRRER MJ, ROSENBERG RK et al. p53: a frequent target for genetic abnormalities in lung cancer. Science 1989; 246: 491-494. https://doi. org/10.1126/science.2554494

[28] VIKTORSSON K, DE PETRIS L, LEWENSOHN R. The role of $\mathrm{p} 53$ in treatment responses of lung cancer. Biochem Biophys Res Commun 2005; 331: 868-880. https://doi. org/10.1016/j.bbrc.2005.03.192

[29] LIM SO, KIM H, JUNG G. p53 inhibits tumor cell invasion via the degradation of snail protein in hepatocellular carcinoma. FEBS Letters 2010; 584: 2231-2236. https://doi. org/10.1016/j.febslet.2010.04.006

[30] WANG SP, WANG WL, CHANG YL, WU CT, CHAO YC et al p53 controls cancer cell invasion by inducing the MDM2mediated degradation of Slug. Nat Cell Biol 2009; 11: 694704. https://doi.org/10.1038/ncb1875

[31] POWELL E, PIWNICA-WORMS D, PIWNICA-WORMS H. Contribution of p53 to Metastasis. Cancer Discovery 2014; 4: 405. https://doi.org/10.1158/2159-8290.CD-13-0136 